\title{
Indium Tin Oxide Nanoparticle-modified Glassy Carbon Electrode for Electrochemical Sulfide Detection in Alcoholic Medium
}

\author{
Md. Abdul AzIZ, ${ }^{* \dagger}$ Rakan AlMADI, ${ }^{* *}$ and Zain Hassan YAMANI* \\ * Center of Excellence in Nanotechnology (CENT), King Fahd University of Petroleum and Minerals, \\ KFUPM Box 5040, Dhahran 31261, Saudi Arabia \\ **Chemical Engineering Department, The University of Manchester, UK
}

\begin{abstract}
An electrochemical sensor based on an indium tin oxide nanoparticle (ITONP)-modified glassy carbon electrode (ITONP/ GCE) was developed for the detection of sulfides in alcoholic medium. The ITONP/GCE was prepared by simply dropping the aqueous solution of ITONP on the GCE surface and then drying at $40^{\circ} \mathrm{C}$. The homogeneous distribution of ITONP on the GCE surfaces was confirmed by recording the camera photographs and field emission scanning electron microscopic images. The ITONP/GCE was further characterized with energy dispersive X-ray spectrometry, X-ray photoelectron spectroscopy and X-ray diffractometry to identify the surface chemical composition and crystallinity. The ITONP/GCE showed much better electrocatalytic properties toward sulfide electro-oxidation than that of bare GCE electrodes. The obtained amperometric detection limit of sulfide in alcoholic solutions of sodium acetate was $0.3 \mu \mathrm{M}$. The enhanced sensitivity, good selectivity and high stability are promising features of the ITONP/GCE for sulfide sensing.
\end{abstract}

Keywords ITO nanoparticle, glassy carbon electrode, electrode modification, drop-drying method, electrocatalytic properties, sulfide sensing, amperometry

(Received November 27, 2017; Accepted January 16, 2018; Published May 10, 2018)

\section{Introduction}

Sulfur, especially in the form of a sulfide, is an abundant naturally occurring element that is part of many systems ranging from oil and gas reservoirs to environmental and biological systems around the globe. ${ }^{1-5}$ It is also produced as a byproduct during the production of alcohol by the fermentation of sugar present in raw agricultural products. The same yeast, which is responsible for the production of alcohol from sugar, can convert the present sulfur species (e.g. elemental sulfur, sulfate or sulfite) into sulfide. ${ }^{6-9}$ Ethanol or mixed alcohols are also prepared from syngas using molybdenum sulfide or alkaline metal molybdenum sulfide as the catalyst. ${ }^{10-12}$ Sometimes, hydrogen sulfide $\left(\mathrm{H}_{2} \mathrm{~S}\right)$ gas is flowed to maintain the constant activity of the catalyst. ${ }^{10,11}$ As a result, the alcohols prepared from syngas using molybdenum sulfide often contain sulfide. ${ }^{10,12}$

Sulfide anion is highly toxic to living organisms. ${ }^{1-4}$ Sulfide exist as $\mathrm{S}^{2-}$, hydrosulfide $\left(\mathrm{SH}^{-}\right)$and $\mathrm{H}_{2} \mathrm{~S}$ depending on the nature of the environment. ${ }^{2,13}$ It is soluble in both alcoholic and aqueous media. ${ }^{2,13}$ The potent threat to humans is that exposure to sulfide anions causes several diseases, such as neurological and cardiovascular disorders, conjunctivitis, photophobia, corneal bullae, tearing, pain and blurred vision..$^{1-4,14,15}$ It can even cause sudden death. In addition, combustion of alcoholic fuels (alternative fuels) or hydrocarbon fuels such as gasoline and natural gases that contain sulfur, including sulfide, generates sulfur dioxide $\left(\mathrm{SO}_{2}\right)$ and this contributes to air pollution, posing

† To whom correspondence should be addressed.

E-mail: maziz@kfupm.edu.sa adverse health and environmental risks. ${ }^{16-18}$ High sulfur content in fuels reduces the efficiency of the catalytic reaction of a system, and leads to emission of air pollutants such as nitrous oxides, carbon monoxide, and volatile organic compounds in the environment. ${ }^{17,18}$ Moreover, it reduces the half-life of motor vehicles since it is a corrosive agent for the metallic parts of vehicles. Also, alcohols are used as common solvents for a variety of practical applications, such as synthesis and isolation of molecules including drugs. However, the quality of the product partially depends on the purity of the alcohols. As a result, the contaminant, sulfide, should be removed; i.e., desulfurization is required before using alcohol as a fuel or solvent. The above discussion indicates that the detection of sulfides from alcohols or alcoholic solutions is very important to maintain the quality of the alcohol for various purposes such as protecting the environment and health, increasing the longevity of motor engines, and maintaining the quality of the products.

There are various methods for the detection of sulfides in alcohols, such as chromatographic, ${ }^{10,12}$ spectrophotometric flow injection, ${ }^{9}$ chemiluminescence, ${ }^{6}$ and colorimetric techniques. ${ }^{6,19}$ However, most of these classical methods are laboratory-based due to their large size and requirement of long analysis time and use of toxic chemicals. In addition, these methods require skilled personnel and expensive instrumentation, which increases the overall cost of analysis. Their high cost is an obstacle for routine analysis of sulfide. On the contrary, the electrochemical method is a well-known method in the analytical community for portable, cheap, selective and sensitive detection of analytes. But the success of the electrochemical sensor greatly depends on the nature of the working electrode. Precious metals (e.g. gold, platinum, and palladium) and the glassy 
carbon electrode (GCE) are common working electrodes used in electrochemistry. The use of precious metallic electrodes for sulfide sensing is not wise due to the high affinity of the precious metal to sulfurous species, which leads to severe poisoning of the electrode surface. ${ }^{14,15}$ This also applies to the bare GCE. ${ }^{20}$ In addition, the GCE has poor electrocatalytic properties toward many electroactive molecules. This poor stability and the low electrocatalytic properties should be improved for the fabrication of a sensitive sensor.

To improve the electrocatalytic properties of a base electrode, immobilization of nanocatalysts, such as gold, silver, platinum, copper, palladium, indium tin oxide (ITO) nanoparticle (NPs), carbon nanotubes or graphene, is a common approach nowadays. ${ }^{14,21-32}$ Moreover, modification of a substrate electrode with a suitable nanocatalyst could help to overcome the surface poisoning effect. However, the cost of the sensor based on a nanomaterial-modified electrode partially depends on the immobilization method, as well as cost of the catalyst. To immobilize the nanocatalyst on electrode surfaces, various methods such as electrochemical, pre-functionalization of nanomaterials or electrodes with different linker molecules, sputtering and successive annealing, thermal driven attachment, seed mediated growth and drop-drying of nanoparticle solution on the electrode have been reported..$^{14,21-32}$ We recently immobilized the ITONPs on (3-aminopropyl)triethoxysilane functionalized ITO electrode surface for improving the electrocatalytic properties towards sulfide oxidation in aqueous solutions. ${ }^{14}$ The fabricated ITONP-modified electrode was employed to detect sulfide in aqueous solutions (not in alcoholic solutions). From this, we anticipated that the ITONP may play a role as an electrocatalyst for sensing the sulfide in alcoholic medium also. However, the electrode modification method should be simplified for its wide application. Among the developed methods, the direct drop-drying method is a straightforward, simple and economic approach. However, the use of the drop-drying method is limited due to the frustrating aggregation of the NPs in certain locations on the solid substrate. ${ }^{33,34}$ It could be meaningful in terms of cost minimization and also be considered as straightforward method if some nanomaterials can be deposited with homogeneous distribution on bare electrode surfaces by drop-drying without requiring highly sophisticated techniques. Overall, the aim of this paper is modification of the bare GCE with a nanocatalyst using a simple and straightforward method for improving the electrocatalytic properties and stability of the GCE electrode toward selective and sensitive sulfide sensing in alcoholic medium.

Here we report a drop-drying method for the immobilization of ITONP with homogeneous distribution on a bare GCE for electrochemical detection of sulfide in alcoholic medium. The modified electrode was characterized with field emission scanning electron microscopy (FESEM), camera, energy dispersive X-ray spectroscopy (EDS), X-ray photoelectron spectroscopy (XPS), and X-ray diffractometery (XRD). The voltammetric analysis ascertains that the ITONP-modified GCE (ITONP/GCE) electrode possesses higher electrocalytic properties toward sulfide electro-oxidation in alcoholic media compared to that of a bare GCE. The responses of the resulting ITONP/GCE sensor to various sulfide concentrations were determined by carrying out amperometry (i-t) experiments. The detection limits obtained using amperometry was $0.3 \mu \mathrm{M}$. Moreover, this optimized electrode was highly selective toward sulfide in the presence of common interfering species. Furthermore, we proved that the ITONP/GCE electrode is more stable in alcoholic solutions of sulfide compared to bare GCE and $\mathrm{Au}$ electrodes.

\section{Experimental}

\section{Reagents and chemicals}

Colloidal $18 \mathrm{~nm}$ ITONPs $\left(20 \mathrm{wt} \%\right.$ in $\left.\mathrm{H}_{2} \mathrm{O}\right)$, sodium acetate, sodium sulfide nonahydrate $\left(\mathrm{Na}_{2} \mathrm{~S} \cdot 9 \mathrm{H}_{2} \mathrm{O}\right)$, sulfur $(\mathrm{S})$, benzene, xylene and phenol were purchased from Sigma-Aldrich. The GC disk electrode with diameter of $3 \mathrm{~mm}$ was purchased from $\mathrm{CH}$ instruments. The GC plate electrode was offered by Prof. Munetaka Oyama, Kyoto University, Japan. Anhydrous mixed alcohol composed of specially denatured alcohol (200 proof, SDA 3A, containing methanol) $95 \%$ and 5\% isopropyl alcohol was purchased from J. T. Baker Chemical Co. The stock solution of $30 \mathrm{mM} \mathrm{Na}_{2} \mathrm{~S}$ was made via ultra-sonication of the appropriate amount of $\mathrm{Na}_{2} \mathrm{~S} \cdot 9 \mathrm{H}_{2} \mathrm{O}$ in the mixed alcohol. Similarly, an inert electrolyte solution of $20 \mathrm{mM}$ sodium acetate was made by dissolving an appropriate amount of sodium acetate in the mixed alcohol.

\section{Preparation of the ITONP/GCE}

The ITONP/GCE was prepared by the drop-drying method. Initially, the supplied ITONPs colloid was diluted to $2 \mathrm{mg} \mathrm{mL}^{-1}$ with deionized water. Both the GC disk electrode and GC plate electrode were polished with $0.05 \mu \mathrm{m}$ alumina/water slurry (Buehler, USA) on a polishing cloth to a mirror-like finish, followed by sonication and then rinsed with distilled water. After drying the polished substrate, $20 \mu \mathrm{L}$ of the ITONP solution was dropped on the GC disk electrode and GC plate separately and successively dried in an oven at $40^{\circ} \mathrm{C}$ to obtain the ITONP-modified GCE. Next, we utilized the ITONPmodified GC disk electrode as the working electrode while the ITONP-modified GC plate electrode was used for surface characterization.

\section{Instrumentation}

All electrochemical measurements were performed using a CHI (760E) electrochemical workstation (http://www. chinstruments.com). Bare gold, bare GCE and ITONP/GCE electrodes were employed as the working electrodes, $\mathrm{Ag} / \mathrm{AgCl}$ was employed as the reference electrode, and a platinum wire was employed as the counter electrode. All electrochemical experiments were carried out at room temperature without deaeration of the solution. FESEM images were recorded using a field emission scanning electron microscope (TESCAN LYRA 3, Czech Republic). Energy dispersive X-ray spectra (EDS) were collected using an Xmass detector (Oxford Instruments) equipped on the Lyra3 TESCAN FESEM. An XPS equipped with an $\mathrm{Al}-\mathrm{K \alpha}$ micro-focusing $\mathrm{X}$-ray monochromator (ESCALAB 250Xi XPS Microprobe, Thermo Scientific, USA) was employed for the chemical analysis of the electrode material. X-ray diffraction patterns (XRD) of the ITONP/GCE were acquired using a Rigaku MiniFlex X-ray diffractometer (Japan) equipped with $\mathrm{Cu}-\mathrm{K} \alpha 1$ radiation.

\section{Results and Discussion}

Morphological, chemical compositional and structural characterization of the ITONP/GCE

The surface of the bare GCE (Fig. 1A) is smooth whereas the surface of ITONP/GCE (Fig. 1B) is characteristic of NPs. Figure 1B clearly shows the presence of densely gathered semispherical NPs along with some cubic NPs. The details of the shape and sizes of ITONPs were discussed in our earlier report. ${ }^{14}$ The low magnified view of ITONP/GCE (Fig. 1C) and 

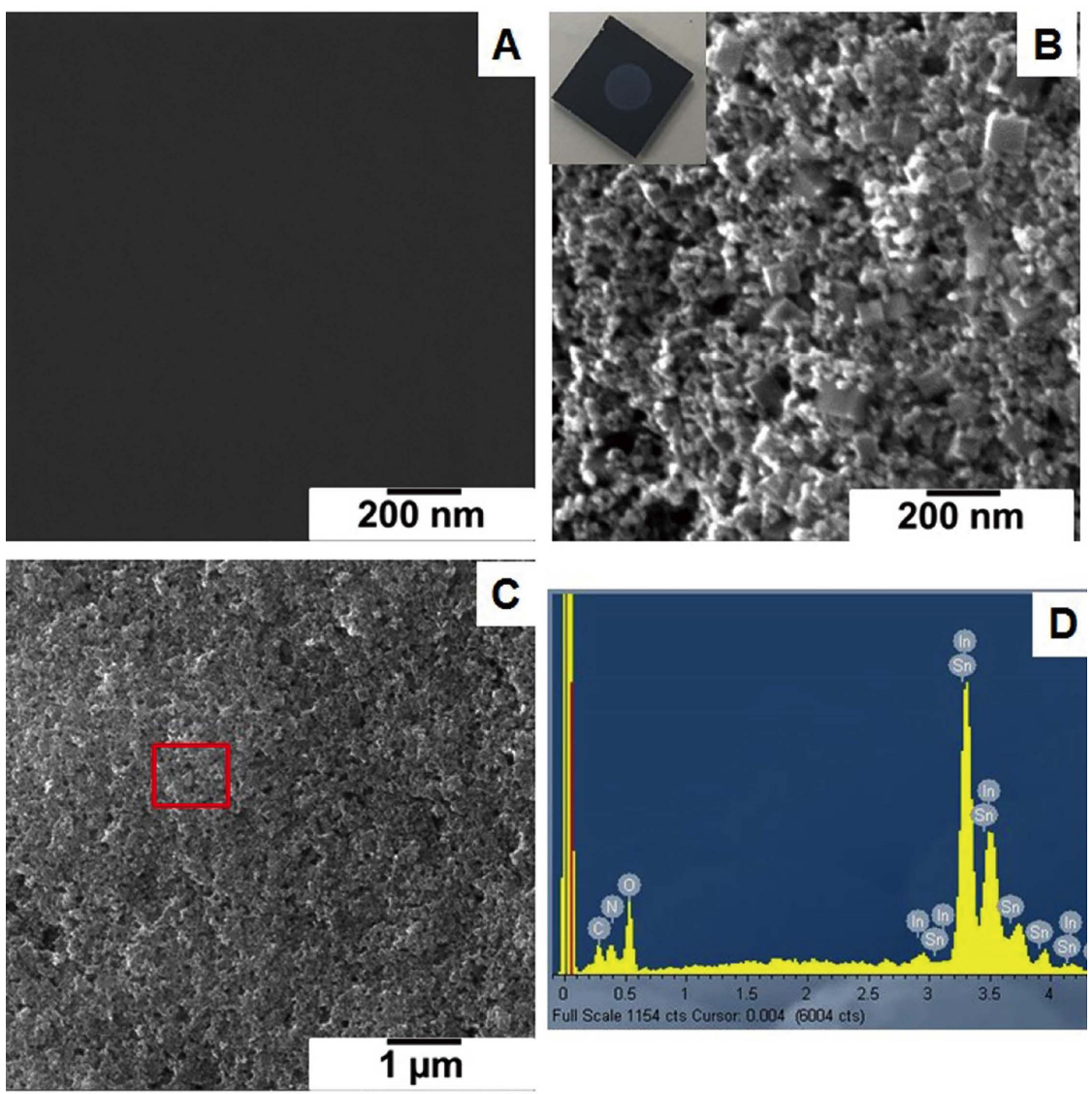

Fig. 1 FESEM images of the bare GCE (A) and ITONP/GCE (B). (C) Low magnification image of B. Inset of (B) is the corresponding photograph of the ITONP/GCE. (D) EDS spectrum of the red marked area of $(\mathrm{C})$.

corresponding photograph (bluish white circle in inset of Fig. 1B) clearly indicate the homogeneous distribution of NPs on the GCE surface even though the ITONP/GCE was prepared by direct drop-drying of $20 \mu \mathrm{L}$ aqueous solution of $2 \mathrm{mg} \mathrm{mL}^{-1}$ ITONPs on the GCE. This result is quite different from the formation of ITONP film on an ITO electrode. To obtain the homogeneous film of ITONP on the ITO electrode, surface functionalization with linker molecules was a prerequisite. The exact reason for the homogenous distribution of ITONP on the GCE surface is not well understood yet. The "coffee-stain" effect due to convective macroscopic flow into the contact line creates the main barrier for homogenous distribution of NPs by the drop-drying method. ${ }^{33}$ Perhaps, the coffee-stain effect was minimized for the present case due to the yet unidentified properties of aqueous solution of ITONP and surface properties of the GCE. Interestingly, the obtained diameter of the ITONP film on the GC plate electrode is around $3 \mathrm{~mm}$, which is similar to that of the used GC disk electrode. As a result, similar film of ITONP was found when $20 \mu \mathrm{L}$ aqueous solution of the $2 \mathrm{mg}$ $\mathrm{mL}^{-1}$ ITONPs were drop-dried on the GC disk electrode of $3 \mathrm{~mm}$ diameter.

Figure 1D shows the EDS spectrum of the ITONP/GCE. The spectrum shows the presence of $\mathrm{In}, \mathrm{Sn}, \mathrm{O}$ and $\mathrm{C}$ peaks, as expected. However, the reason for the small peak for $\mathrm{N}$ in the EDS spectrum is not identified. The chemical composition of the ITONP/GCE was further characterized with XPS (Fig. 2A). The XPS shows the major peaks for In, Sn and O, as marked in Fig. 2A. The carbon peak became almost invisible due to the high intensity of major peaks even though the ITONP/GCE
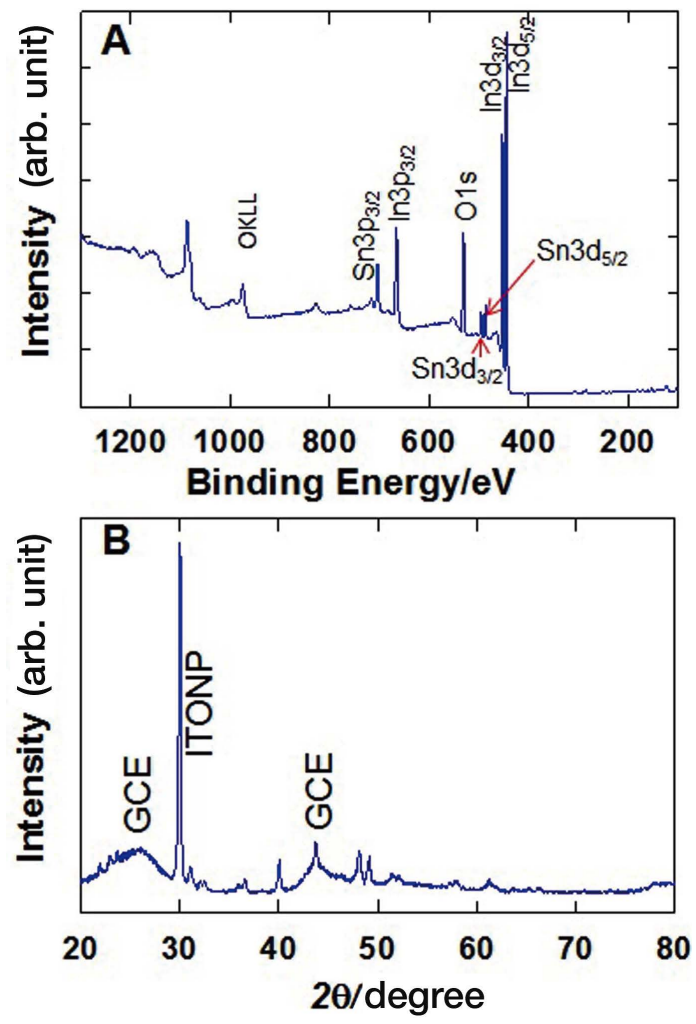

Fig. 2 XPS spectrum (A) and XRD patterns (B) of the ITONP/GCEs. 

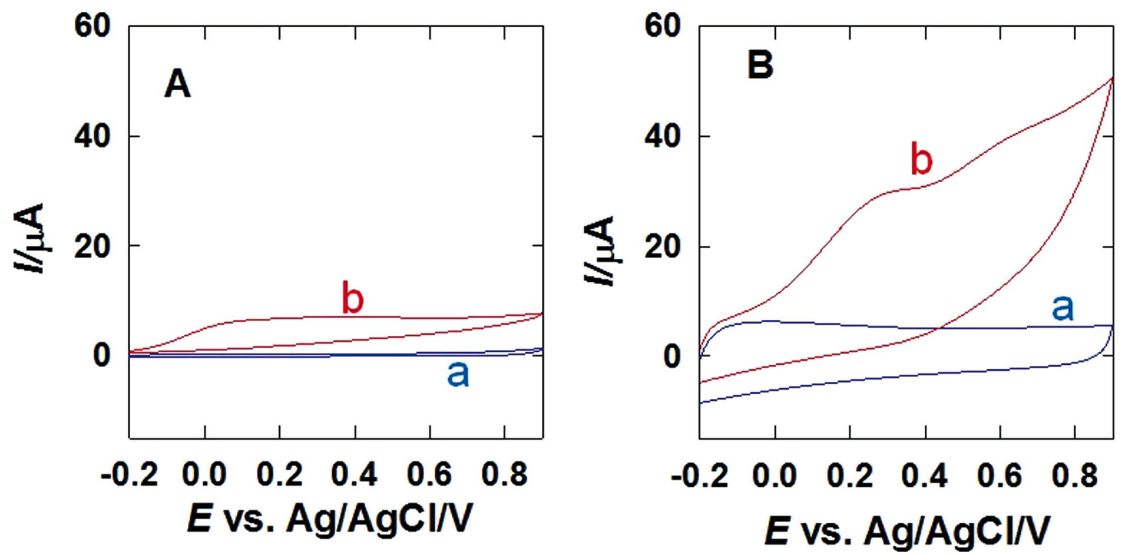

Fig. $3 \mathrm{CVs}$ of the bare GCE (A) and ITONP/GCEs (B) in the mixed alcohol containing $20 \mathrm{mM}$ sodium acetate in the absence (a) and presence (b) of $1 \mathrm{mM} \mathrm{Na}_{2} \mathrm{~S}$. Scan rate: $50 \mathrm{mV} / \mathrm{s}$.

contains carbon as a base electrode. It is to be noted that the detection depth of the XPS is only a few nanometers. It indicates that the ITONP is densely gathered on the GCE surface and there is almost no exposed surface of the GCE. This finding correlates with the FESEM analysis (Figs. 1B and 1C).

Figure 2B shows the XRD spectrum of the ITONP/GCE. The peaks at $25.94^{\circ}$ and $43.74^{\circ}$ are related to the GCE electrode, which was confirmed by recording the XRD of the bare GCE using the identical experimental condition (data is not shown). The major peaks for ITONP appeared at $30.02^{\circ}$, which corresponds to the (222) crystal plane of indium oxide, indicating the cubic symmetry of the crystals. ${ }^{35}$

\section{Electrocatalytic properties}

We evaluated the enhanced electrocalaytic properties of the ITONP/GCE by recording the cyclic voltammetry $(\mathrm{CV})$ of the bare GCE (Fig. 3A) and ITONP/GCE (Fig. 3B) in the absence (curves a of Fig. 3) and presence (curves b of Fig. 3) of $1 \mathrm{mM}$ $\mathrm{Na}_{2} \mathrm{~S}$ in the mixed alcoholic solution of $20 \mathrm{mM}$ sodium acetate. By comparing curve a with curve $b$ of both electrodes, it can be concluded that both the bare GCE and ITONP/GCE can oxidize the sulfide in alcoholic media. However, the obtained sulfide oxidation current with the ITONP/GCE is much higher than that obtained with the bare GCE, although the starting oxidation potential $(-0.15 \mathrm{~V})$ as well as peak potential for sulfide electrooxidation $(\sim 0.38 \mathrm{~V})$ are almost similar. It is also observed that the sulfide electroxidation current at the ITONP/GCE increased with increasing the potential after peak potential, whereas it remained nearly constant in the case of the bare GCE electrode. The reason for this might be oxidation at peak potential zone of sulfide ion to elemental sulfur, and that was further oxidized to sulfite ion $\left(\mathrm{SO}_{3}{ }^{2-}\right)$ or sulfate ion $\left(\mathrm{SO}_{4}{ }^{2-}\right)$ with as increase of the potential on the ITONP surface. ${ }^{15}$ The above discussion concludes that overall, the ITONP/GCE possesses higher electrocalytic properties compared to that of a bare GCE. The improved electrocatalytic properties of the ITONP/GCE might arise from (i) the high surface area of the ITONP/GCE, as higher background/capacitive current of the ITONP/GCE (Fig. 3Ba) was obtained compared to that of the bare GCE (Fig. 3Aa) and (ii) unique electrocatalytic properties of ITONPs. ${ }^{14,36}$

\section{Amperometric determination of sulfide concentration}

Depending on the voltammetric result, amperometric experiments were carried out to obtain the limit of detection and
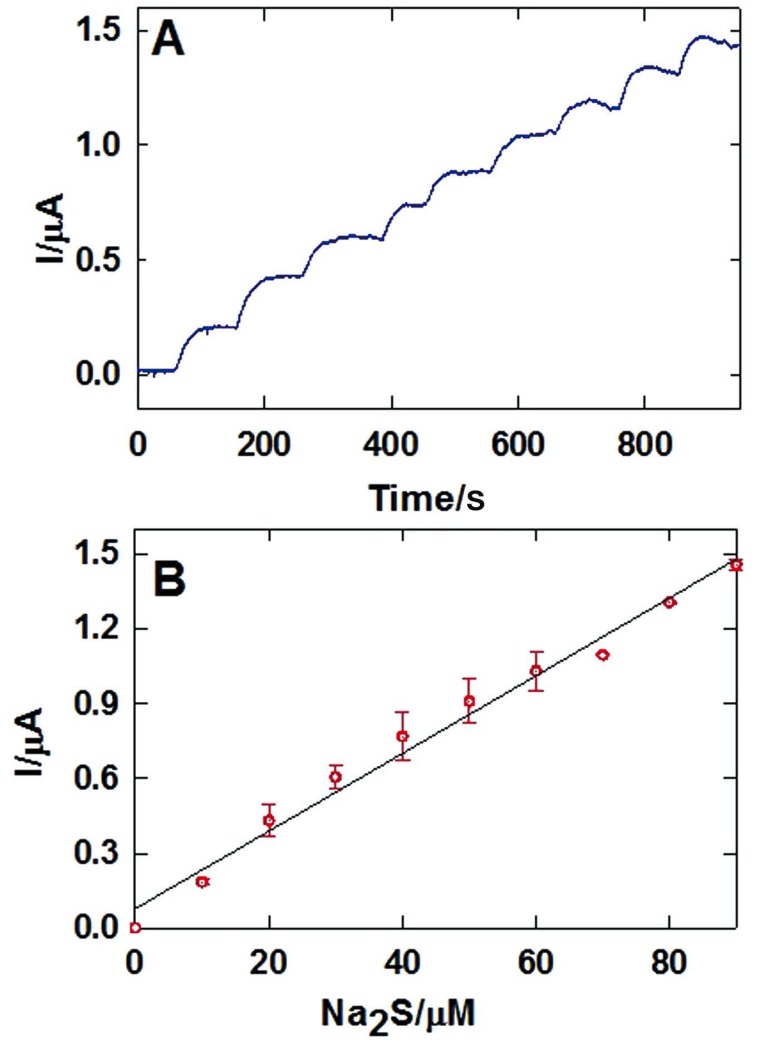

Fig. 4 (A) Amperogram of the ITONP/ITO electrode in mixed alcohol containing $20 \mathrm{mM}$ sodium acetate at $+0.3 \mathrm{~V}$ during the successive addition of $10 \mu \mathrm{M}$ of $\mathrm{Na}_{2} \mathrm{~S}$. (B) The corresponding calibration plot.

sensitivity of the ITONP/GCE-based sulfide sensor. Figure 4A shows a current-time (i-t) curve for the ITONP/GCE-based sensor at $0.3 \mathrm{~V}$ upon successive addition of $10 \mu \mathrm{M}$ sulfide in the mixed alcohol containing $20 \mathrm{mM}$ sodium acetate. The ITONP/ GCE generated a distinct and sensitive signal for each addition of sulfide in the range of $10-90 \mu \mathrm{M}$ as seen in Fig. 4A. The relationship between the generated current (after subtracting the mean of the zero sulfide response) and the sulfide concentration was found to be linear over the range $10-90 \mu \mathrm{M}$ (Fig. 4B). The corresponding linear regression equation is 


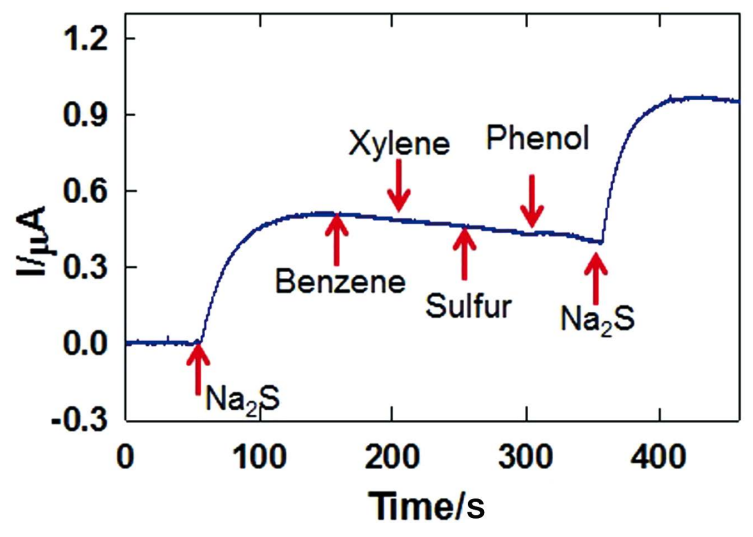

Fig. 5 Amperometric responses of the ITONP/GCE in the mixed alcohol containing $20 \mathrm{mM}$ sodium acetate at $+0.3 \mathrm{~V}$ upon successive addition of $25 \mu \mathrm{M} \mathrm{Na} 2 \mathrm{~S}, 30 \mu \mathrm{M}$ benzene, $30 \mu \mathrm{M}$ xylene, $30 \mu \mathrm{M}$ sulfur, $30 \mu \mathrm{M}$ phenol and $25 \mu \mathrm{M} \mathrm{Na} 2 \mathrm{~S}$ again.

$I(\mu \mathrm{A})=0.0156[$ Sulfide $](\mu \mathrm{M})+0.0775\left(R^{2}=0.98\right)$. The limit of detection of the ITONP/GCE-based sensor for sulfide was calculated to be $0.3 \mu \mathrm{M}$. This obtained detection limit is much lower than that obtained with ITONP-modified 3-aminopropyltrimethoxysilane-functionalized ITO electrodes or bare ITO electrodes for the detection of sulfide from aqueous sulfide solutions in our earlier report. ${ }^{14,15}$

\section{Selectivity}

Elemental sulfur, benzene, and xylene are often present to some extent in alcohol or alcoholic media. They can potentially interfere with the sulfide signal. The selectivity test of the developed sulfide sensor was performed against the above mentioned species. Figure 5 shows the i-t curve of the ITONP/ GCE at $0.3 \mathrm{~V}$ upon consecutive spiking with sulfide, benzene, xylene, elemental sulfur, phenol and once more with sulfide. After addition of $25 \mu \mathrm{M}$ sulfide, a well-defined signal was observed. Further consecutive spiking with $30 \mu \mathrm{M}$ each of benzene, xylene, elemental sulfur, and phenol did not alter the initial sulfide signal remarkably. However, a well-defined signal was observed again upon spiking of another aliquot with $25 \mu \mathrm{M}$ sulfide. This result reflects the high selectivity of the developed sensor for sulfide. The good selectivity of sulfide sensing at the ITONP/GCE might originate from poor and high electrocalytic properties of the ITONP/GCE electrode for tested interferents and sulfide ion, respectively.

\section{Stability}

The ITONP/GCE exhibited stability with high electrocatalytic activity while sensing for sulfide. Figure 6Aa presents the amperometric signal for a $200 \mu \mathrm{M}$ sulfide solution at $0.3 \mathrm{~V}$ for a 1000 -s period. The response stayed almost unaltered during the total experiment, reflecting that the sulfide and/or its oxidation products did not suppress the catalytic properties of the ITONP/GCE. For comparison studies, we recorded the amperograms of $200 \mu \mathrm{M}$ sulfide in the identical experimental condition with the bare GCE as well as with the gold electrode. Upon addition of $200 \mu \mathrm{M}$ sulfide, an oxidation signal was generated, but decreased significantly over time for both cases. After $1000 \mathrm{~s}, 38.85 \%$ and $14.5 \%$ of the original signal remained in the case of the bare GCE and gold electrode, respectively, indicating fast surface passivation or poisoning of the both electrode surfaces. ${ }^{14,20}$ These experimental results clearly demonstrate that the developed ITONP/GCE is significantly
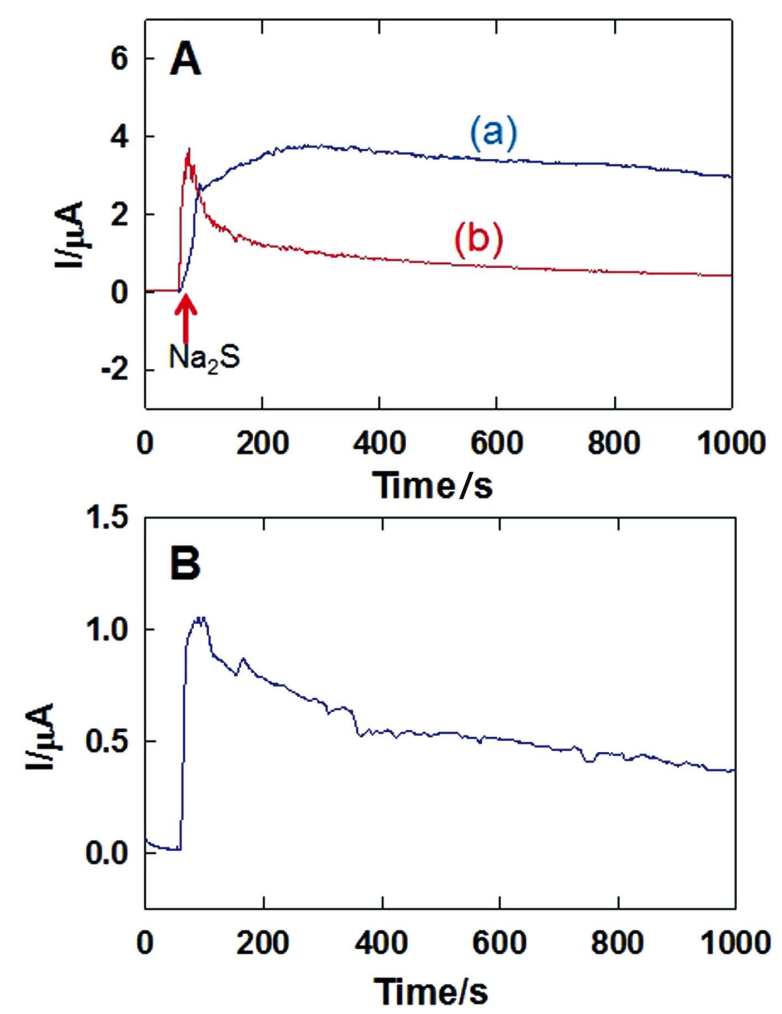

Fig. 6 Amperometric responses of the ITONP-GCE A (a), Au disk electrode A (b), and bare GCE (B) to a $200 \mu \mathrm{M} \mathrm{Na} \mathrm{N}_{2} \mathrm{~S}$ solution in the mixed alcohol containing $20 \mathrm{mM}$ sodium acetate at $+0.3 \mathrm{~V}$ over a long period of time.

more stable compared to other widely used electrodes. The reason for the high stability of the ITONP/GCE is the low affinity of ITONPs to sulfide molecules and their oxidized forms of sulfides. ${ }^{14}$

\section{Conclusions}

This study developed a novel method for the preparation of a uniform ITONP-modified GCE by the drop-drying method for highly sensitive, selective and stable detection of sulfide ions in alcoholic media. The electrodes showed significantly higher electrocatalytic properties toward sulfide oxidation than that of the bare GCE electrode. The detection limit of the sensor using the amperometric method was $0.3 \mu \mathrm{M}$ of sulfide. The ITONP/ GCE showed good selectivity and was not susceptible to interference by common interferents. The ITONP/GCE also showed higher stability in the sulfide environment than the bare GCE and Au electrode. The overall performance quality of the novel ITONP/GCE proved to be excellent, and the electrodes were suitable for the analytical determination of sulfide concentrations in a variety of alcohol related applications.

\section{Acknowledgements}

The authors acknowledge funding support from King Abdulaziz City for Science and Technology (KACST) through the Science \& Technology Unit at King Fahd University of Petroleum \& Minerals (KFUPM): Project No.14-ENV332-04, as part of the National Science, Technology and Innovation Plan. 


\section{References}

1. Work Place Health and Safety Bulletin, Hydrogen Sulphide at the Work Site, prepared by Government of Alberta, 2010, http://work.alberta.ca/documents/WHS-PUB-CH029.pdf.

2. M. K. Amosa, I. A. Mohammed, and S. A. Yaro, NAFTA, 2010, 61, 85 .

3. Toxicological Profile for Hydrogen Sulfide, prepared by US Department of Health and Human Services, Agency for Toxic Substances and Disease Registry, 1600 Clifton Road NE, Mailstop F-32 Atlanta, Georgia 30333, 2006, http:// www.atsdr.cdc.gov/toxprofiles/tp114.pdf.

4. Y. Chen, R. Kirankumar, C. Kao, and P. Chen, Electrochim. Acta, 2016, 205, 124.

5. T. Xu, N. Scafa, L. Xu, S. Zhou, K. A. Al-Ghanem, S. Mahboob, B. Fugetsu, and X. Zhang, Analyst, 2016, 141, 1185.

6. S. Park, J. Microbiol. Biotechnol., 2008, 18, 1550.

7. C. E. Butzke and S. K. Park, J. Microbiol. Biotechnol., 2011, 21, 519.

8. Production of Hydrogen Sulfide During Fermentation, prepared by Scott Laboratories, Scott Laboratories, 2220 Pine View Way, Petaluma, CA, http://www.scottlab.com/ uploads/documents/Production $\% 20$ of $\% 20$ Hydrogen $\% 20$ Sulfide.pdf.

9. C. R. Silva, V. A. F. Barros, L. C. Basso, and E. A. G. Zagatto, Talanta, 2011, 85, 1703.

10. R. Andersson, $\mathrm{PhD}$ thesis entitled, "Catalytic Conversion of Syngas to Higher Alcohols over MoS2-based Catalysts", KTH Royal Institute of Technology, School of Chemical Science and Engineering, Department of Chemical Engineering and Technology, Stockholm, Sweden 2015, https://www.diva-portal.org/smash/get/diva2:778216/ FULLTEXT01.pdf.

11. M. Konarova, F. Tang, J. Chen, G. Wang, V. Rudolph, and J. Beltramini, ChemCatChem, 2014, 6, 2394.

12. R. Andersson, M. Boutonnet, and S. Järås, J. Chromatogr. A, 2012, 1247, 134.

13. A. V. Kurzin, A. N. Evdokimov, V. S. Golikova, and O. S. Pavlova, J. Chem. Eng. Data, 2010, 55, 4080.

14. M. A. Aziz, W. Mahfoz, M. N. Shaikh, M. H. Zahir, A. AlBetar, M. Oyama, D. Theleritis, and Z. H. Yamani, Electroanalysis, 2017, 29, 1683 and references therein.

15. M. A. Aziz, M. Sohail, M. Oyama, and W. Mahfoz, Electroanalysis, 2015, 27, 1268.

16. T. Lenior, Literature thesis chemistry entitled, "Analytical Techniques for the Determination of Sulphur Components in Flue Gas, Fuel Gas and Natural Gas", Department of Analytical Chemistry \& Applied Spectroscopy, Vrije Universiteit, Amsterdam, 2009, https://www.asap.nl/wpcontent/uploads/2013/08/analytical-techniques-for-thedetermination-of-sulphur.pdf.

17. Sulfur Levels in Gasoline \& Diesel, http://www.transportation. alberta.ca/Content/docType57/Production/Sulphur-Levels. pdf.

18. K. Zhang, J. Hu, S. Gao, Y. Liu, X. Huang, and X. Bao, Energy Policy, 2010, 38, 2934.

19. T. E. Acree, E. P. Sonoff, and D. F. Splittstoesser, Appl. Microb., 1971, 22, 110.

20. N. S. Lawrence, R. P. Deo, J. Wang, Anal. Chim. Acta, 2004, 517, 131.

21. M. M. Alshalalfeh, M. Sohail, T. A. Saleh, and M. A. Aziz, Aust. J. Chem., 2016, http://dx.doi.org/10.1071/CH16072.

22. M. A. Aziz, S. Patra, and H. Yang, Chem. Commun., 2008, 4607.

23. S. K. Majil, A. K. Dutta, D. N. Srivastava, P. Paul, A. Mondal, B. Adhikary, and U. Adhikary, J. Nanosci. Nanotechnol., 2013, 13, 4969.

24. A. Kawde M. Aziz, N. Baig, and Y. Temerk, J. Electroanal. Chem., 2015, 740, 68.

25. A. Kawde and M. A. Aziz, Electroanalysis, 2014, 26, 2484.

26. M. A. Aziz and A. Kawde, Microchim. Acta, 2013, 180, 837.

27. M. A. Aziz and H. Yang, Bull. Korean Chem. Soc., 2007, 28,1171

28. S. Wu, Q. He, C. Tan, Y. Wang, and H. Zhang, Small, 2013, 9, 1160.

29. M. A. Aziz and M. Oyama, Adv. Mater. Res., 2014, 995, 125.

30. M. R. Akanda, M. Sohail, M. A. Aziz, and A. Kawde, Electroanalysis, 2016, 28, 408.

31. Q. Wang, Z. Tang, L. Wang, H. Yang, W. Yan, and S. Chen, Chem. Select, 2016, 1, 6044.

32. M. I. Ahmed, M. A. Aziz, A. Helal, and M. N. Shaikh, J. Electrochem. Soc., 2016, 163, D277.

33. M. Majumder, C. S. Rendall, J. A. Eukel, J. Y. L. Wang, N. Behabtu, C. L. Pint, T. Liu, A. W. Orbaek, F. Mirri, J. Nam, A. R. Barron, R. H. Hauge, H. K. Schmidt, and M. Pasquali, J. Phys. Chem. B, 2012, 116, 6536.

34. R. Duggal, F. Hussain, and M. Pasquali, Adv. Mater, 2006 , 18, 29.

35. M. A. Aziz, M. I. Ahmed, M. Qamar, and M. N. Shaikh, J. Mater. Sci: Mater. Electron., 2016, 27, 10325.

36. M. Wei, R. Huang, and L. Guo, J. Electroanal. Chem., 2012, 664, 156. 